\title{
AUTOTRADUCTION ET FIGURES DU DÉDOUBLEMENT DANS LA PRODUCTION DE NANCY HUSTON ${ }^{1}$
}

\section{Valeria Sperti}

\section{L'ambiguïté linguistique}

Nancy Huston, d'origine canadienne anglophone, est une polyglotte tardive. Son début littéraire est à Paris, où elle s'installe vers 1973, avec Les Variations Goldberg, en 1981, récit composé en français tout comme ses deux romans suivants, Histoire d'Omaya de 1985 et Trois fois septembre de 1989. L'écrivaine explique son choix de composer dans la langue acquise, à première vue surprenant, par sa biographie. Les raisons seraient psychologiques: blessée par l'abandon de sa mère, qui quitte le toit familial quand Nancy est âgée de six ans pour une vie nouvelle sans emmener ses enfants avec elle, l'auteure trouve dans le "théâtre de l'exil" (Huston 1999, p. 30), avec sa langue nouvelle, décontaminée des traumatismes d'enfance, une réparation, une planche de salut :

Pour ma part j'ai commencé par écrire en français afin d'échapper à ma langue maternelle [...] [j'ai pris] mon envol grâce à la liberté et à la légèreté que me conférait le français, l'illusion qu'elle m'octroyait de n'avoir pas d'enfance, pas d'inconscient, pas de racines, pas de déterminisme... (Huston 2007a, p. 154).

Toutefois, ces affirmations jubilatoires ne font pas long feu: la "rassurante étrangeté" (Huston 1981, p. 199) de l'exilée volontaire, bien née et bien insérée dans l'effervescence intellectuelle de la capitale, se transforme en malaise, physique et psychologique. L'écriture en français se change en écran reflétant une imitation littéraire que l'auteure perçoit comme inauthentique et distante. La langue maternelle est comparée à un muscle atrophié: seule une réhabilitation pourra la sortir de cette "position linguistique presque perverse" (Huston 2001, p. 23). Le traitement s'avère passer par la traduction, encryptée dans le roman comme un thème narratif: dans Trois fois septembre, Solange, une jeune fille française vivant aux États-Unis, transpose simultanément à sa mère le journal en

\footnotetext{
${ }^{1}$ La troisième section de cet article inédit (Instruments des ténèbres et ses doubles autobiographiques) a été présentée au colloque "Nancy Huston: le soi multiple/Nancy Huston: The Multiple Self", Université Paris 3Sorbonne nouvelle, 8-9 juin 2012.
} 
SPERTI - Autotraduction et figures du dédoublement...

anglais de son amie américaine disparue. C'est une mise en abyme textuelle de l'acte d'écriture et de traduction, représentés comme contemporains et inséparables. Et encore, nous constatons la présence d'un thème destiné à devenir un topos: la narration parcourt et s'inscrit dans le sillage d'un autre texte, intime, dans ce cas un dernier témoignage écrit dans une langue autre que celle de l'amie-lectrice française. Sans doute ce stade intermédiaire a-t-il été important pour la composition du roman suivant. En fait, après trois années de silence romanesque, de 1990 à 1993, passées à traduire, réviser et éditer les versions anglaise et française de Plainsong, roman du retour aux sources - le premier rédigé en anglais - l'écrivaine entame une réflexion profonde sur son identité bi-culturelle et bi-linguistique. La publication contemporaine de Plainsong et Cantique des plaines en 1993 (Huston 1993a et 1993), avec sa suite de polémiques suscitées par l'attribution du Prix du Gouverneur général du Canada à la version française - perçue par quelques éditeurs et journalistes québécois comme un roman traduit, donc disqualifié parce qu'il n'est pas l'original - relie indissolublement la carrière littéraire de l'écrivaine à l'autotraduction. Dans Plainsong Paula, l'héroïne, écrit de son appartement montréalais le récit de la vie de son grand-père Paddon dans les grandes plaines de l'Alberta, à partir du manuscrit que Paddon lui-même lui confie à sa mort. Le choix de situer l'histoire dans le contexte bilingue montréalais et dans la région anglophone de ses origines, qualifie ce roman comme un retour littéraire au pays natal. La structure binaire, où les mots de la narratrice découlent des mots contenus dans "de vieilles pages noircies" (Huston 1993, p. 9) que son grand-père lui a léguées, est ici particulièrement significative. Créer une fiction à partir des mots de l'autre devient, non seulement une charpente récurrente dans l'œuvre romanesque hustonienne, mais une figure du double qu'il est possible, selon notre opinion, de relier à la projection dans le texte du dédoublement linguistique que l'autotraduction rend explicite. Le lecteur est ainsi introduit dans l'atelier de l'écrivaine et regarde, de ce point de vue intérieur, la fiction et les langues qui la composent.

Nancy Huston a pratiqué "l'autotraduction consécutive" (Grutman 2009, p. 260) avant la publication du roman attaqué au Québec, précisément à l'occasion de la publication de sa version anglaise d'Histoire d'Omaya, sortie en français en 1985 et en traduction deux ans plus tard (Huston 1987). Toutefois, à la suite de l'attribution du prix prestigieux, Nancy Huston se trouve dans l'inconfortable position de devoir se justifier, 
SPERTI - Autotraduction et figures du dédoublement...

dans son pays, le Canada, d'avoir écrit dans sa langue maternelle, l'anglais. "It was as if the prodigal son had come home and his father said to him 'Go fuck yourself'. I, of course, had imagined something quite different" (Huston in Davey 2004, p. 3). Pour répondre aux malentendus rocambolesques de ses détracteurs, elle souligne dans les épitextes le côté créatif de la translation interlinguistique. Tout en se rendant compte que "c'est cette coexistence inconfortable, en moi, de deux langues et de deux façons d'être qui me rend le plus profondément canadienne. Elles ne veulent surtout pas se réunir [...]; elles tiennent à se critiquer [...] elles revendiquent toute l'ambiguïté de leur situation" (Huston 1993b, p. 261), elle se consacre - âme et corps — à des réflexions sur le bilinguisme, sur l'identité et ses déclinaisons linguistiques entre idiome maternel et idiome acquis et sur la part qu'occupent les deux langues dans sa création. Ces notations paraissent, sous forme d'essais, entre 1993 et 1997: Pour un patriotisme de l'ambiguïté, En français dans le texte,Festins fragiles, Le déclin de l'identité (Huston 1993b, 1994, 1994a, 1997a) enracinent, moyennant l'expérience personnelle et la réflexion théorique, le processus autotraductif dans l'acte de création. L'écrivaine, d'ailleurs, se réclame d'une vision herméneutique de la traduction comme forme première de l'acte créatif, qui consiste à transposer le monde en mots :

[L'auteur] écrit pour agrandir le monde, pour en repousser les frontières. Il écrit pour que le monde soit doublé, aéré, irrigué, interrogé, illuminé par un autre monde, et qu'il devienne habitable. Ce faisant l'écrivain traduit (Huston 2007, p. 153).

Dans ces essais, elle insiste sur sa pratique particulière de l'autotraduction. Chaque roman est composé dans une langue (dictée par le contexte narratif, par sa racontabilité), puis traduit dans l'autre avant d'être publié. L'avantage est évident: les deux versions dialoguent, estompant les différences entre original et copie; des modifications - de rythme, de ton, de style - sont apportées tant à l'original qu'à la version traduite. Cette pratique de "l'autotraduction simultanée" (Grutman 2009, p. 260), commencée de manière fortuite avec Cantique, est présentée ensuite par l'auteure comme une habitude, sans doute après les polémiques du Prix, acquérant une connotation défensive. La traduction anglaise d'Histoire d'Omayade 1987et celle des Variations Goldberg parue en 1996, une quinzaine d'années après la publication française et à la suite du succès grandissant de l'auteure, constituent 
SPERTI - Autotraduction et figures du dédoublement...

une exception à la règle. Une exception qui montre que, dans cette phase cruciale de sa production littéraire, Nancy Huston aspire à être publiée et reconnue aussi en tant qu'écrivaine anglophone. La pratique autotraductive se configure aussi comme une barrière défensive érigée contre les détracteurs de l'auteure qui ne la considèrent ni québécoise, ni francophone et vise à lénifier le malaise inhérent à son ambiguïté bilingue (Sperti 2013, p. $384)$.

\section{Nancy Huston et sa fratrie littéraire: Romain Gary et Samuel Beckett}

La réflexion sur l'autotraduction se déploie aussi dans deux petits livres à l'appartenance générique floue, consacrés l'un à Romain Gary - Tombeau de Romain Gary en 1995 - l'autre à Samuel Beckett - Limbes/Limbo, publié en édition bilingue en 2000 que l'écrivaine investit du rôle de génies tutélaires de son ambiguïté linguistique. Les deux partagent la condition de "Corps étranger dans la littérature française" (Huston 2001, p. $25)$; l'impureté dérivant de leur statut identitaire et linguistique les relie à Nancy Huston par une fratrie littéraire réunissant les écrivains déplacés, disloqués : "Il ne me quittera plus" (p. 26) dit-elle du premier; le second est désigné de "mon frère, mon pied" (Huston 2000, p. 25). Bien que différents, les deux textes relèvent d'une matrice commune. Le premier est un hommage conventionnel, au moins dans le titre; le deuxième une pirouette critique et mimétique sur les œuvres de Beckett. Les deux élaborent par transformation, imitant et parodiant le système littéraire de ces écrivains autotraducteurs, la revendication de l'ambiguïté irréductible de l'in-between linguistique. Dans la reconstruction biographique de Huston, le fantasme identitaire de Romain Gary et ses dédoublements à travers les pseudonymes et les langues seraient la cause de son suicide. La leçon de Beckett est évidente dans sa présentation typographique: les deux versions en français et en anglais, publiées en regard, définissent l'autotraduction comme une pratique littéraire qui soude, lénifiante dans sa composition parallèle, mais dynamique dans ses différences, constituant dans leur dialogue une réponse définitive aux partisans de l'original, de l'authentique. Les deux textes, par leurs formes intermédiaires entre création littéraire et critique, oscillent entre l'hommage passionné et la parodie désopilante. Ils mettent en pratique le "déracinement de la théorie" (Huston 2001, p.15) souhaité par l'écrivaine et une représentation du double susceptible de répondre aux questionnements identitaires et 
SPERTI - Autotraduction et figures du dédoublement...

linguistiques. Il ne s'agit plus de représenter par le mode binaire de l'antinomie, où une langue exclut, suit ou précède l'autre dans le processus créatif, car les appartenances et les identités découlent des reflets et des mélanges entre ces cultures différentes. Dans Tombeau de Romain Gary et Limbes/Limbo la compréhension de l'autre, plus précisément son appréhension - sous forme d'identité, de langue, de culture - passe par le sens étymologique du latin cum-prehendere, où l'écriture englobe l'autre ${ }^{2}$. Dans Limbes/Limbo, le flux de conscience et le démantèlement des mécanismes ordinaires du langage - "la langue charriant son non-sens" (Klein-Lataud 2004, p. 45) - amplifiés par la présence de deux idiomes, thématise et inscrit l'appropriation de l'autre dans le texte et par l'écriture. La modalisation de la voix narrative, la théâtralisation de la narration et les références intertextuelles communes sont autant de jalons posés pour la réflexion identitaire et scripturaire hustonienne. Elle se ressource à l'impact énorme que l'écrivain russe et l'écrivain irlandais ont exercé sur son imaginaire. Du dernier elle affirmera, à distance de plusieurs années, qu' “il est là, avec moi, en moi” (Huston 2005, p. 71). Cette duplicité transposée d'une langue à l'autre, en acceptant les 'trahisons' inévitables de toute traduction, acquiert le statut d'autobiographème. Pour mieux comprendre ce qui se passe dans la fiction, il faut revenir à la production romanesque qui suit Cantique des plaines.

\section{Instruments des ténèbres et ses doubles autobiographiques}

En 1996, peu de temps avant Limbes/Limbo - dont une première version avait été rédigée en 1997, éditée en version monolingue l'année suivante et publiée en bilingue en 2000 (Danby 2004)- Huston publie Instruments des ténèbres (1996), un roman dont la transposition anglaise paraîtra l'année suivante (1997). Écrit entre 1994 et 1995 - à courte distance des amertumes causées par le prix canadien - le roman connaît un succès important en France, couronné par le Prix du Livre Inter, qui consacre Nancy Huston comme une écrivaine française, ce qui ne manque pas de susciter en elle quelques perplexités. Le roman fait alterner, chapitre après chapitre, l'histoire de deux femmes, Nadia qui habite entre Manhattan et la campagne environnant New York dansles années soixante et Barbe, une jeune fille du Berry au temps de Louis XIV, qui subit un procès pour

\footnotetext{
${ }^{2}$ Pour les thèmes du féminin, de la procréation et de la création littéraire, cf. Huston 1990 et MacDonald 2003, p. 7-16.
} 
SPERTI - Autotraduction et figures du dédoublement...

sorcellerie. L'auteure déclare avoir rédigé le roman passant de l'anglais du journal intime, où Nadia évoque ses difficultés procréatives, au français du roman historique, dont la narratrice est toujours Nadia, racontant les péripéties de la paysanne naïve et démunie.

Le ton de ces déclarations est jubilatoire, pareil à l'enthousiasme de celui qui a trouvé la quadrature du cercle: "J'ai composé l'histoire alternée des deux femmes en passant de l'anglais au français, chapitre après chapitre. Tous les jours, je me reposai d'une langue sur l'autre et y puisai un regain d'énergie" (Huston in Gazier 1997). Compte tenu de l'alternance linguistique, il apparaît évident que ce roman manifeste dans sa composition même l'ambiguïté linguistique définie plus haut. Mais ce qui est encore plus intéressant, c'est qu'il encrypte dans la fiction des thèmes sur lesquels l'écrivaine réfléchit à l'époque; souvent la configuration du double qui est exposée dans la narration cache des consonances autobiographiques. En fait, cette première expérience de rédaction bilingue, occasionnée par les deux contextes hétérolinguistiques du roman, s'accompagne d'autres éléments significatifs. Le plus évident est celui de la gémellité: Barbe a un frère jumeau, Barnabé. Leur naissance, les séparant en tant qu'individus, scinde l'élément féminin de l'élément masculin: s'agit-t-il d'une métaphore de deux langues qui s'alternent dans le roman? Le journal de Nadia - composé en anglais - révèle une femme en mal d'identité et s'intitule Scordatura, qui indique une composition musicale écrite pour un instrument à cordes dont les accords ont été modifiés. S'agit-t-il d'une métaphore du processus de rédaction du roman, où l'alternance énonciative entre la première personne (le journal) et la troisième (le récit) marque le changement de langue, connotant une dissonance linguistique? Le récit de Barbe s'intitule Sonate de la Résurrection, comme la composition baroque d'Heinrich Biber $^{3}$ qui pour son exécution fait appel à la Scordatura, soulignant ainsi les intersections qui multiplient les perspectives de lecture et les pistes d'associations: littérature et musique, procréation et création bilingue.

L'éditeur français refuse de publier le roman dans sa double partition linguistique; Nancy Huston est contrainte de traduire en français le journal de Nadia et en anglais le récit de Barbe, afin de publier les deux textes, séparément, en version monolingue (Raoul 2001, p. 448-449). L'enchâssement textuel, en tant que stratégie de composition, juxtapose deux continents - l'Amérique du Nord et l'Europe - et deux époques - la contemporanéité floue

\footnotetext{
${ }^{3}$ Sur la conjonction musico-littéraire dans ce roman, cf.Arroyas 2007.
} 
SPERTI - Autotraduction et figures du dédoublement...

de Nadia-Nada et le XVIII ${ }^{\mathrm{e}}$ siècle - en faisant ressortir leur distance linguistique et culturelle. La fiction traduit ainsi l'identité divisée de Nancy Huston, accentuée à l'époque par la polémique de Cantiques.

La réappropriation de la langue anglaise comme langue de rédaction, déjà entamée dans Cantique des plaines, accompagnée de l'imbrication entre texte et métatexte qui se poursuit dans Instruments, constitue la fictionnalisation - en tant que mise à distance du réel - de l'autobiographème du dédoublement identitaire. Nadia affirme: "Moi aussi j'ai besoin du dédoublement, de la duplicité. Pas de visions sans division. Je ne cesse de comparer, combiner, séduire, traduire, trahir. J'ai le cœur et le cerveau fendus, comme les sabots du Malin. Anglais, français" (Huston 1996, p. 20). Les deux récits - le Carnet et la Sonate - procèdent parallèlement, avec une alternance rigoureuse. De plus, ils se recoupent: le premier garde les traces de la rédaction du second. Le daimôn de Nadia dans le Carnetà la fois conscience morale, surmoi rigoureux et ange gardien - exerce une fonction d'intermédiaire entre les deux dimensions de Nadia et met en dialogue les apparitions magiques du récit fictionnel de la Sonate avec sa voix-off dans le journal. L'univers baroque de Barbe se prête à cette narration en télescopage qui a une fonction heuristique importante. Par la mise en texte des expériences de la jeune paysanne, Nadia appréhende et dépasse la détresse de sa solitude, la mort de son frère jumeau ${ }^{4}$ et la séparation d'avec sa famille (Holmes 2010, p. 35-40). La valence thérapeutique, que l'écriture à cheval entre deux langues a pour Nadia, est un autre autobiographème que Nancy Huston a explicité à maintes reprises dans ses essais et dans les épitextes et qui est repris dans la fiction du journal intime d'Instruments des ténèbres. L'écriture du Carnet agit comme la scordatura en musique: elle déplace et déstructure les instances conflictuelles de Nadia pour recomposer ses différents aspects, y compris son identité bilingue.

Dans les pages de grand-titre de l'édition française du roman, rien n'est dit de la composition bilingue originale, ni de la traduction. L'édition pour les pays anglophones ne mentionne que le titre français juxtaposé à celui en anglais, sans aucune précision ultérieure. Toutefois, au niveau du texte, dans les deux versions, la présence de termes appartenant à d'autres langues est révélatrice. Riche en emprunts de termes musicaux

\footnotetext{
${ }^{4}$ Le jumeau de Nadia était mort à la naissance: "On était deux là-dedans. Je vécus neuf mois avec mon frère. C'est lui qui fut étranglé dans la confusion innommable de notre naissance, alors que je m'en tirai avec la moindre égratignure" (Huston 1996, p. 65).
} 
SPERTI - Autotraduction et figures du dédoublement...

italiens et allemands, latins et yiddish, le roman étale des références lexicales et culturelles anglophones et francophones qui se disputent le terrain. Dans la version francophone, à partir du titre, une citation du Macbeth shakespearien ${ }^{5}$, jusqu'aux vers des chansons de l'afro-américain Nat King Cole, de nombreuses références appartiennent à la culture anglophone. Dans The Scordatura Notebook, la version originairement composée en anglais, plusieurs mots français jalonnent la prose - des adjectifs, des citations, des proverbes, des expressions- autant de traces que l'écrivaine-narratrice laisse de l'autre langue qui l'habite, brisant le monolinguisme en tant qu'horizon mental. C'est notamment dans les deux versions du journal de Nadia que l'autre idiome est présent, représentant textuellement ce qui se passe dans la composition (Shread 2009; Klein-Lataud 1996). C'est en effet la première fois qu'autotraduction et rédaction se juxtaposent dans le même roman (El Nossery 2007, p. 394).

La plasticité de chaque langue qui accueille des éléments d'autres idiomes jusqu'aux sons de la nature que Barnabé imite, les traduisant du ton de sa voix - manifeste une configuration du dédoublement comme compréhension de l'autre en soi. Il est évident que la transposition linguistique qui a concerné les deux textes pour rédiger les versions monolingues respectives se situe en dehors de la narration et ne peut pas constituer un autobiographème au sens propre. Toutefois le Carnet Scordatura, à la date du 6 février, contient un propos intéressant de Nadia sur la traduction en tant que translation du monde par l'écriture et que nous rappelle l'essai de 2007 déjà cité:

Tout est traduction désormais. Mes livres sont des traductions, par exemple : des tentatives maladroites, bâclées, pour transcrire ce que m'a révélé mon daimôn. L'original n'existe pas. L'original est comme le paradis: perdu par définition (Huston 1996, p. 106).

Le dédoublement représenté comme une crise de la subjectivité est une préoccupation constante dans les essais de l'auteure, notamment dans Lettres parisiennes (Huston et Sebbar 1986) et Nord Perdu (Huston 1999). Dans Instruments des ténèbres le choix du décor baroque confère à cette crise un cadre historique précis. La complémentarité de Barbe et de Barnabé est poreuse: devenu adulte, ce dernier ressemble à une femme. De même, la première est déféminisée par le dur travail et la détresse alimentaire qui la rendent

\footnotetext{
${ }^{5}$ Acte I, scène 3 où Banquo met en garde Macbeth sur le danger de la prophétie des sorcières.
} 
SPERTI - Autotraduction et figures du dédoublement...

semblable à un jeune adolescent. C'est sur cette ressemblance - où se fondent qualités masculines et féminines - que se construit l'improbable échange d'identité final qui soude, par la solidarité et le sacrifice, leur amour fraternel.

Tant Barbe dans la Sonate que Nadia dans le Carnet ont des frères jumeaux: celui de Barbe se sacrifie pour sauver sa sœur; celui de Nadia, mort à la naissance, se transforme en daimôn, témoin parfait et double surmoïque de la narratrice. Leur dialogue remplit le Carnet et Nadia se libère de lui après avoir terminé d'écrirela Sonate, après avoir ressuscité son identité d'écrivaine.

Le Carnet est le double fictionnel du travail de rédaction de Nancy Huston, une ouverture du laboratoire de l'écrivaine, qui laisse percer le regard du lecteur dans le processus de composition et de traduction. Nadia enlève de son prénom la lettre "I" - en anglais le pronom représentant l'identité personnelle - pour souligner qu'elle est Nada, le néant; elle récupère la lettre identitaire par l'écriture, une fois le roman achevé ${ }^{6}$. Les deux premières lettres de son prénom, NA, incorporées dans celui de Barbe, forment le nom de son frère Barnabé. Mais NA sont aussi les deux lettres du prénom de l'écrivaine. Nadia aussi, comme Nancy Huston, part en Europe à la recherche d'une page blanche où inscrire sa nouvelle identité (Holmes 2010, p. 37). Dans ces jeux de télescopage, le Carnet s'impose comme le double fictionnel de la rédaction accomplie par Nancy Huston et comme une mise en abyme de la composition romanesque de la Sonate, corroborant l'importance de l'écriture dans la définition identitaire tant de Nancy que de Nadia. Celle-ci affirme :

J'en ai fini avec les jamais et les toujours, les tout et les rien. Dorénavant j'embrasserai les mixtures, les choses mitigées, et me contenterai de morceaux de perfection [...]. L'Enfer et le Paradis sont tous les deux ici sur Terre. Nulle part ailleurs. Nulle part ailleurs (Huston 1996, p. 249).

Dans l'évolution de la production hustonienne des années qui suivent la publication de Cantiques de plaines, les trois ouvrages que nous avons pris en compte - Tombeau de Romain Gary,Limbes/Limbo et Instruments des ténèbres - jouent un rôle important. À travers l'œuvre de Romain Gary, l'écrivaine expérimente le dédoublement non plus dans les termes d'opposition irréductible, mais de contiguïté poreuse, lieu de rencontre et de

\footnotetext{
${ }^{6}$ À ce moment, Nadia peut se libérer de son daimôn: "Il me semble que vous êtes devenu, comment dire... de trop" (Huston 1996, p. 248).
} 
SPERTI - Autotraduction et figures du dédoublement...

coexistence d'identités et de langues différentes, issues de l'instabilité politique et des séparations familiales et géographiques.

Par Limbes/Limbo, pièce de virtuose, Huston rend hommage à l'écrivain irlandais en l'exorcisant par le pastiche bilingue. Le travail de l'auteure, dont l'écriture traverse l'œuvre de Romain Gary et Samuel Beckett, est l'occasion d'une mise au point nécessaire pour trouver sa voix personnelle, pour transformer sa détresse identitaire en richesse littéraire. Les autobiographèmes encryptés dans Instruments des ténèbres indiquent que Nancy Huston n'est pas dupe de sa fiction "immersive" (Holmes 2010, p. 88). Le roman de Nadia et de Barbe affiche une surcharge de make-believe qui est la marque de la fiction hustonienne; les différentes configurations du dédoublement montrent que l'auteure reconnaît dans la richesse et dans le plaisir du texte fictionnel la part de subterfuge qui consiste à incarner dans des personnages romanesques ses propres fantômes, ses propres doubles linguistiques et identitaires.

\section{Les lignes de faille des langues et la danse noire de l'autotraduction}

Dans Lignes de faille (Huston 2006) les histoires des quatre personnages - tous âgés de six ans et tous rejetons de générations précédentes appartenant à la même souche familiale - se déroulent pour la plupart en Amérique du nord. L'écrivaine a composé ce roman en anglais et l'a autotraduit en français. La narration est riche d'effets d'accumulation, car tous les membres de cette famille sont liés par des secrets familiaux et tous vivent des moments importants de la grande histoire, du conflit irakien et du massacre de Sabra et Chatila, à la deuxième guerre mondiale et au programme d'adoptions forcées pratiqué par les Nazis. Aux failles familiales se superposent les failles linguistiques. Chaque personnage affronte, aime ou déteste la langue de l'autre: allemand, arabe, hébreu, polonais et anglais. Kristina-Erra, la mère fondatrice de la famille, a oublié sa langue maternelle et sa recherche des origines, à la suite d'innombrables péripéties, est douteuse tout comme celle de Nancy Huston a été littérairement trompeuse. Sol déteste la langue et la nourriture de l'Allemagne. Randall apprend l'hébreu et l'arabe, mais il apprend aussi, et à ses dépens, la faille qui les sépare. Quant à Kristina-Erra (dont l'identité nominale reste problématique), elle croit que sa langue maternelle est l'allemand, puis le polonais, pour découvrir à la fin de la guerre que c'était l'ukrainien, une langue à jamais perdue, 
SPERTI - Autotraduction et figures du dédoublement...

puisqu'elle est adoptée au Canada, où elle apprend l'anglais. Les difficultés linguistiques des personnages du roman nous semblent une métaphore, mutatis mutandis, du flottement “doublement mi-lingue" (Huston 1986, p. 77) que l'écrivaine a subi lorsque l'abandon de sa mère l'a livrée à l'arbitraire linguistique et identitaire?

Danse noire (Huston 2013) entrelace trois destins individuels et générationnels d'une même famille, représentés sous forme d'ébauche de scénario de film, stratagème métanarratif mettant à nu le processus d'écriture. Le roman a été composé en anglais, en français, quelques passages en joual. La traduction de l'anglais est en bas de page et puisqu'elle se réfère souvent à l'anglais parlé par les Amérindiensde langue algonquine, Nancy Huston a décidé d'utiliser la parlure québécoise des années cinquante. Dans la version anglaise, prévue pour l'automne 2014 aux États-Unis mais pas au Canada, ce seront les dialogues en français à être traduits en note. Ce sous-titrage, parfois lassant, est lié au cadre narratif cinématographique: "C'est le livre le plus pervers linguistiquement que j'ai jamais fait et je ne recommencerai pas! Mais il fallait faire ça une fois dans ma vie" affirme l'écrivaine (Boisvert 2013). Pour la première fois, l'autotraduction partielle en bas de page se juxtapose à l'autre langue, celle du roman. La multiplication des idiomes, des registres linguistiques en français, anglais, anglo-canadiens et cri, force le lecteur à un vaet-vient exténuant entre les notes et le texte (Beaujour 2015). Danse noire est présenté au lecteur comme unroman total pour la mixité des langues et pour l'autotraduction en prise directe. Mais cette dernière, reléguéeaux notes, a-t-elle encore la même valence que dans les ouvrages précédents que nous avons examinés? Est-elle encore un procédé créatif d'autoréflexion, un jeu affirmatif de création et le lieu d'une incontournable recherche autobiographique? Nous n'en sommes pas sûre.

\footnotetext{
${ }^{7}$ Kristina-Erra choisit de chanter: sa voix vibrante, qui la rendra célèbre, exprime ses émotions sans paroles. Nancy Huston partage-t-elle l'ambition d'Erra-Kristina, ce chant asémantique, reflet d'une unité perdue où la traduction est superflue?
} 
SPERTI - Autotraduction et figures du dédoublement...

\section{Bibliographie}

ARROYAS, Frédérique. Diabolus in musica: La "Sonate de la Résurrection" de Heinrich Biber, arme de détournement dans "Instruments des ténèbres" de Nancy Huston. L'Espritcréateur, v. 47, n. 2, 2007. p. 88-100.

Beaujour, Elizabeth Klosty. Nancy Huston's Danse Noire. L2 Journal. UC Davis, 2015. http://escholarship.org/uc/item/5zx9b5pj Consulté mai 2014.

BOISVERT, Richard. Danse noire de Nancy Huston: le rythme du cœur. LeSoleil, 6 octobre 2013. http://www.lapresse.ca/le-soleil/arts-etspectacles/livres/201310/05 /01-4696945-danse-noire-de-nancy-huston-le-rythme-du-coeur.php. Consulté mai 2014.

DANBY, Nicola. The Space Between Self-translator: Nancy Huston's Limbes.La Linguistique, v. 40, n. 1, 2004. p. 83-96.

DAVEY, Frank. Big, Bad and Little Known: The Anglophone-Canadian Nancy Huston. In: DVOŘÁK, Marta \& KOUSTAS, Jane. Vision/Division: l'ouvre de Nancy Huston. Ottawa: Presses de l'Université d'Ottawa, 2004. p. 3-21.

EL NOSSERY, Névine. L'étrangeté rassurante de la "bi-langue" chez Abdelkébir Khatibi et Nancy Huston. Contemporary French and Francophone Studies, v. 11, n. 3, 2007. p. 389-397.

GAZIER, Michèle; LAVAL, Martine \& BOUCHEZ, Emmanuelle. Français dans le texte, entretien avec Nancy Huston. Télérama, 1997. http://www.telerama.fr/livre/ francais-dans-le-texte,69949.php. Consulté mai 2014.

GRUTMAN, Rainier. Self-translation. In: BAKER, Mona\& SALDANHA, Gabriela (eds.), Routledge Encyclopedia of Translation Studies. London: Routledge, 2009. p. 258-260.

HOLMES, Diana. No common places: exile as loss and gain in the work of Nancy Huston and other writers from elsewhere. Dalhousie French Studies, n. 33, 2010. p. 33-42.

HUSTON, Nancy. Les Variations Goldberg. Paris: Seuil, 1981. (1981) La rassurante étrangeté. In: HUSTON, Nancy. Désirs et réalités. Arles/Montréal : Actes Sud/Leméac, 1995. p. 199-208.

Histoire d'Omaya. Paris : Seuil, 1985. 
The Story of Omaya. London: Women's Press Fiction, 1987.

Trois fois septembre. Paris: Seuil, 1989.

Journal de la création. Paris: Seuil, 1990.

Cantique des plaines. Arles/Montréal: Actes Sud/Leméac, 1993.

. Plainsong. Toronto: HarperCollins, 1993a.

- Festins fragiles. Liberté, v. 36, no 6 (216), diciembre 1994. p. 7-15

. (1994) Pour un patriotisme de l'ambiguïté. Notes autour d'un

voyage aux sources. Saint- Laurent, Québec: Fides, 1995.

(1994). En français dans le texte. In : HUSTON, Nancy. Désirs et réalités. Arles/Montréal: Actes Sud/Leméac, 1995.p. 263-269.

. Tombeau de Romain Gary, Arles/Montréal: Actes Sud/Leméac, 1995.

. Désirs et réalités. Arles/Montréal: Actes Sud/Leméac, 1995.

. Instruments des ténèbres. Arles/Montréal: Actes Sud/Leméac, 1996.

. Instruments of Darkness. Boston, New York, Toronto, London: Little Brown and Company, 1997.

. Nord Perdu, suivi de Douze France. Arles/Montréal: Actes Sud/Leméac, 1999.

. Limbes/Limbo.Arles/Montréal: Actes Sud/Leméac, 2000.

- (2001) Le Déracinement du savoir, un parcours en six étapes. In: HUSTON, N. Âmes et corps, 2004. p. 13-35.

. (1997a) Le déclin de 1'“identité"? In: HUSTON, N. Âmes et corps. 2004. p. 4965.

. Âmes et corps. Textes choisis 1981-2003, Arles/Montréal: Actes Sud/Leméac, 2004.

. Le Râle vagi: Samuel Beckett. In : HUSTON, N. Professeurs de désespoir. Arles/Montréal: Actes Sud/Lémeac, 2004a.

. Lignes de faille. Arles/Montréal, Actes Sud/Leméac, 2006.

FaultLines. London: Atlantic Books, 2007.

. Traduttore non è traditore. In: LE BRIS, Michel (ed.). Pour une littérature monde. Paris: Gallimard,2007a. p. 151-160.

. Danse noire. Arles/Montréal, Actes Sud/Leméac, 2013. 
et SEBBAR, Leïla. Lettres parisiennes: Autopsie de l'exil. Paris, BernardBarrault, 1986.

KLEIN-LATAUD, Christine. Les voix parallèles de Nancy Huston. TTR: traduction, terminologie, rédaction, v. 9, n. 1, 1996.p. 211-231.

. Langue et lieu d'écriture. In: DVOŘÁK, Marta \& KOUSTAS, Jane. Vision/Division: l'œuvre de Nancy Huston. Ottawa: Presses de l'Université d'Ottawa, 2004. p. 39-48.

MACDONALD, Marylea. L'éthique de la déférence: des récits de vie interstitiels dans Journal de la création de Nancy Huston. Dalhousie French Studies, v. 64, 2003. p. 7-16.

RAOUL, Valérie. L'autre langue fécondatrice: “l'étrangéïté" en soi dans Instruments des ténèbres de Nancy Huston et Possessions de Julia Kristeva. In: LEQUIN, Lucie \& MAVRIKAKIS, Christine (eds.). La francophonie sans frontières: une nouvelle cartographie de l'imaginaire au féminin, Paris: L'Harmattan, 2001. p. 445-454.

SHREAD, Carolyn. Redefining Translation through Self-Translation: The Case of Nancy Huston. French Literature Series, v. 36, n. 1, 2009. p. 51-66.

SPERTI, Valeria. L'ambiguità linguistica di Nancy Huston. In: CECCHERELLI, Andrea et al., Autotraduzione e riscrittura. Bologna: Bononia University Press, 2013. p. 381-394. 